\title{
EMERGENT BEHAVIOUR: THEORY AND EXPERIMENTATION USING THE MANA MODEL
}

\author{
JAMES MOFFAT, JOSEPHINE SMITH, AND SUSAN WITTY
}

Received 25 April 2006; Accepted 15 May 2006

In a previous paper, we introduced the idea of a scale-free system, and applied this to extend the Lanchester equations of industrial age warfare into the information age, corresponding to the shift towards more self-organising force elements in future conflicts. Here, we present evidence from an agent-based model of conflict (MANA) which supports our theoretical analysis.

Copyright (c) 2006 James Moffat et al. This is an open access article distributed under the Creative Commons Attribution License, which permits unrestricted use, distribution, and reproduction in any medium, provided the original work is properly cited.

\section{Introduction}

MANA (Map Aware Nonuniform Automata) is a cellular automata-based simulation model of conflict which has been developed by the New Zealand Defence Technology Agency. It is based on the foundation ideas of complexity and emergence (Lauren and Stephen [12]). It has been developed using as a start point the ISAAC model developed for the US Marine Corps by Illachinski [5]. ISAAC was developed in order to explicitly explore in an experimental fashion the relation between local unit interactions and the resultant emergent properties and behaviour of the overall force.

In the MANA simulation model, a scenario is defined by the battlespace and the characteristics of the two sides (Blue and Red). Each of the two forces is composed of a number of units. These units have the ability to locally sense their environment, and then decide, on the basis of a number of simple algorithms (based on attraction or repulsion to/from neighbouring units of each side and their attraction to their final goal), how to move around the battlespace. Waypoints can also be defined which help to steer the flow of forces in broad general directions.

Over several years we have been working with its main author, Dr. Michael Lauren, through The Technical Cooperation Program (TTCP) on a number of theoretical developments related to MANA. These developments allow an analytical approach to the 
linkage between local clustering assumptions concerning the forces, and the resultant emergent behaviour seen in the battlespace. This emergent behaviour is measured in terms of both expected attrition across the whole simulated conflict and the dynamics of the time series of casualties over simulated time.

Information age forces. The approach is particularly relevant to dispersed, dynamically evolving "information age" scenarios, involving local force clustering and reclustering (a form of local self-organisation). This is in contrast to the force-on-force attrition approach to "industrial age" warfare typified by Lanchester's equations [16]. In [4], Darilek et al. discuss the implications of this thinking for the "information age" US Army, in which autonomous units of force move and cluster across a dispersed battlespace.

Network centric warfare. The linkage between such local self-organisation and global emergent behaviour also lies at the core of ideas such as network centric warfare. This relationship is discussed particularly in the most recent book by Alberts and Hayes [1]. Initial mathematical ideas are also discussed in the book by Moffat [13].

\section{Theoretical analysis}

In a previous paper [14], we considered such a conflict between "Red" and "Blue" forces as an example of a scale-free system, based on the fluid dynamic ideas of Barenblatt [2] and Lauren [9]. We then derived a general relationship for the casualties produced over time in such a conflict. This has the form

$$
E_{0<t<T}\left(\frac{\Delta b}{\Delta t}\right)=E_{0<t<T}\left(-c k^{D / 2} \Delta t^{-(1-D / 2)} r(t)\right)
$$

Here $k$ is the unit effectiveness of a Red agent, $b$ (resp., $r$ ) is the remaining Blue (resp., Red) force strength at time t. $D$ is the fractal dimension of the Red force at time t. $D$ is time-dependent in general as shown by the examples in [14]. $c$ is the constant of proportionality. There is a corresponding relationship for $\Delta r / \Delta t$ in terms of the Blue fractal dimension.

2.1. Correlation in time. We assume that the system is similar to a turbulent flow system where turbulence is multiscaling (in the sense that the effect is recursive at different levels of system resolution). This leads to the assumption of multifractal statistics. This means that the form of expression above applies in a analogous way to all of the moments of the random variable $|(b(t+\Delta t)-b(t)) / \Delta t|$. Thus we have, from the general form of the fractal attrition relationship at (2.1), that

$$
E\left(\left|\frac{b(t+\Delta t)-b(t)}{\Delta t}\right|^{p}\right) \propto \Delta t^{f(D, p)}
$$

where $f$ is a function only of the battlespace casualties fractal dimension $D$ and the order of the moment $p \geq 1$. 
In particular (noting that $\Delta t$ is a constant relative to the expectation $E$ over time $t$ ), the second moment $(p=2)$ should have the relationship

$$
E\left(\left|\frac{b(t+\Delta t)-b(t)}{\Delta t}\right|^{2}\right)=\frac{1}{\Delta t^{2}} E\left(|b(t+\Delta t)-b(t)|^{2}\right) \propto \Delta t^{f(D, 2)},
$$

and hence

$$
E\left(|b(t+\Delta t)-b(t)|^{2}\right) \propto \Delta t^{f(D, 2)+2} .
$$

Initial work by Lauren $[7,10]$ indicates that this relationship is in fact of the form

$$
E\left(|b(t+\Delta t)-b(t)|^{2}\right) \propto \Delta t^{D}
$$

In other words, $f(D, 2)+2=D$. We recall that in $(2.5) b(t)$ is the number of Blue agents at time $t, E$ denotes expectation over time $t$, and $D$ is the fractal dimension corresponding to local clustering of Red agents. In this way, clustering in time of Blue casualties is related to clustering in space of Red (and hence Red casualties) on the battlefield. In the network centric warfare context, we can hypothesise that agile clustering and reclustering of forces by Red (such as swarming effects), measured by the Red fractal dimension $D$, creates clustering in time of Blue casualty effects and hence a particular fractal-based form for the plot of Blue casualties over time.

If we expand the square term on the left-hand side of (2.5), we have

$$
\begin{aligned}
E\left(|b(t+\Delta t)-b(t)|^{2}\right) & =E\left[b(t+\Delta t)^{2}-2 b(t) b(t+\Delta t)+b(t)^{2}\right] \\
& =2 s^{2}-2 \operatorname{corr}(b(\Delta t))
\end{aligned}
$$

where $s^{2}$ is the second moment of the random variable $b(t)$, and $\operatorname{corr}(b(\Delta t))$ is the autocorrelation for the time series of Blue agents $b(t)$ with lag $\Delta t$. We assume (as is usual) that $\Delta t$ is small relative to the complete time span $T$ over which we are taking expectations $E$, with $0 \leq t \leq T$.

Since $s^{2}$ is assumed constant, we can thus see from (2.5) that

$$
\operatorname{corr}(b(\Delta t)) \propto \Delta t^{D}
$$

Considering now a range of values of the lag $\Delta t$, then if a variable such as $\operatorname{corr}(b(\Delta t))$ varies as $\Delta t^{D}$ with $0 \leq D \leq 2$, it follows from $[6,8]$ that

$$
F\{\operatorname{corr}(b(\Delta t))\}(f) \propto|f|^{-(D+1)},
$$

where $F$ is the Fourier transform.

From standard Fourier transform theory, the left-hand side of this expression is just the power spectrum of the time series of Blue agents $b(t)$ :

$$
|F(b)(f)|^{2}=F\{\operatorname{corr}(b(\Delta t))\}(f) \propto|f|^{-(D+1)} .
$$


According to (2.9), the power spectrum should vary with frequency $f$ in accordance with a power law, where the exponent (the exponent $(D+1)$ in $(2.9))$ is proportional to the fractal dimension of the locations of Red agents on the battlefield, corresponding to the agile clustering of these Red agents. Since the Red fractal dimension $D$ lies in the range 0 to 2 , this exponent should lie in the range -1 to -3 . However, the precise form of this exponent will have to be determined experimentally. We assume that a similar power law applies to casualties.

\section{Initial experimental evidence}

In order to derive experimental evidence of these various relationships, an international defence research exchange was set up by the study team under TTCP. Through this process, access was obtained to the Maui High Performance Computer Centre (MHPCC) owned by the US Air Force. This is a supercomputer cluster which allowed, through the US Marine Corps Project Albert process of "data farming" [17], the ability to run models such as MANA many thousands of times across large parameter sets. This was ideal for our purpose of attempting to obtain experimental evidence for our postulated relationships.

3.1. The first term of the fractal attrition relationship. One clear implication of the theory discussed earlier is that the expected rate of Blue attrition $E(|\Delta b / \Delta t|)$ is related to Red unit firepower effectiveness $k$ through the relationship

$$
E\left(\left|\frac{\Delta b}{\Delta t}\right|\right) \propto k^{\alpha}
$$

where $\alpha$ is a power law exponent. A similar relation should also hold between expected Red attrition and Blue unit effectiveness. In the full metamodel at (2.1), this exponent is related to the local clustering between the agents, as measured by the fractal dimension $D$, implying that a noninteger exponent should be anticipated, in general. We analyse this using regression analysis, as part of testing the hypothesis that there is such a power law relationship between expected attrition and unit effectiveness, across a number of sample scenarios.

3.2. Sample scenarios in the MANA simulation model. In MANA, the developers, Lauren and Stephen [11], have noticed four types of emergent behaviour. They are

(i) a Lanchester style battle with two single blocks of force fighting;

(ii) a line of force on force battle which is stable and moves in a wave;

(iii) a line of force on force battle which is unstable;

(iv) swarming behaviour or manoeuvre warfare.

We have used these as a basis for developing a number of scenarios for our experimental investigation. Specifically, we investigated a line of force that is stable and moves in a wave (the "Meet" scenario), swarming behaviour (the "Swarm" scenario), and a Lanchester style battle (the "Lanchester" scenario). The measure of effectiveness that we used for these scenarios was the casualties suffered by the forces, in order to compare with the theoretical metamodel prediction of a power law relationship. 


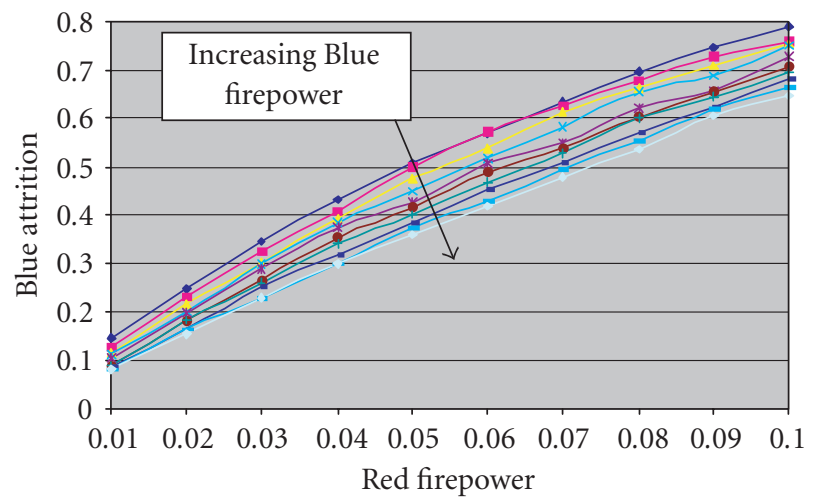

Figure 3.1. Blue attrition against Red unit firepower for different values of Blue unit firepower.

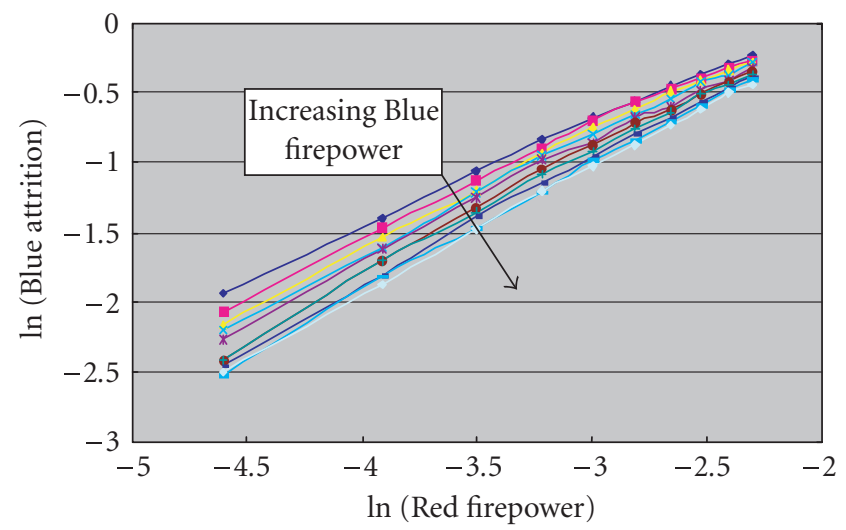

Figure 3.2. Figure 3.1 data shown on a Log-Log plot.

3.3. Experimental results for the "Meet" scenario. Figure 3.1 shows how expected Blue casualties ("attrition") as a proportion of the initial force varies as Red unit firepower is varied along the $x$-axis. In MANA, unit firepower varies between 0 and 100 , and the $x$-axis shows the normalised values. A number of these plots are superimposed, each representing a different value of Blue unit firepower effectiveness. Figure 3.2 shows these same results plotted on a Log-Log scale in order to investigate the power law behaviour predicted by theory. (A power law relationship becomes a straight line on a Log-Log scale.)

Looking at Figure 3.2, we see that the Log-Log plots form very obvious straight lines, indicating that the data has a power law relationship between individual agent firepower and enemy attrition. The gradients of these lines (close to integer values in this case) were found using linear regression and are shown in Table 3.1. They remain stable across the various cases considered. These gradients are the exponent values of the first term of the metamodel at (2.1). 
Table 3.1. Regression line fits for Blue force attrition; "Meet” scenario.

\begin{tabular}{lccc}
\hline Blue firepower & Points & Gradient & Intercept \\
\hline 0.01 & 8 & 0.95 & 0.73 \\
0.02 & 8 & 0.92 & 0.62 \\
0.03 & 8 & 0.9 & 0.54 \\
0.04 & 8 & 0.87 & 0.46 \\
0.05 & 8 & 0.94 & 0.67 \\
0.06 & 8 & 0.87 & 0.5 \\
0.07 & 8 & 0.94 & 0.68 \\
0.08 & 8 & 0.93 & 0.61 \\
0.09 & 8 & 0.89 & 0.5 \\
0.1 & 8 & 0.85 & 0.37 \\
\hline
\end{tabular}

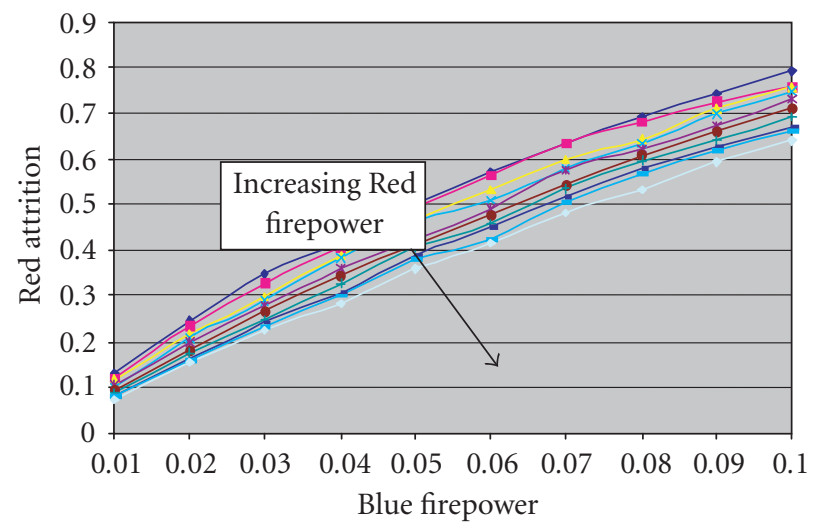

Figure 3.3. Red attrition against Blue unit firepower for different values of Red unit firepower.

Figures 3.3 and 3.4 show the equivalent data for Red casualties.

Table 3.2 shows the linear regression analysis results for the plots in Figure 3.4. Again, the gradient remains reasonably stable across the cases considered. There is evidence here of noninteger gradients, corresponding to fractal effects, as discussed in the earlier theory.

3.4. Implications for the theory. These experimental results for the "Meet" scenario confirm the power law relationship between casualties and unit firepower effectiveness. Similar results were obtained for the "Lanchester" and "Swarm" scenarios. Thus we have established that the first term of (2.1) is of the correct general form for the "Lanchester," "Meet," and "Swarm" scenarios. 
Table 3.2. Regression line fits for Red force attrition; "Meet" scenario.

\begin{tabular}{lccc}
\hline Red firepower & Points & Gradient & Intercept \\
\hline 0.01 & 8 & 0.74 & 1.5 \\
0.02 & 8 & 0.76 & 1.55 \\
0.03 & 8 & 0.8 & 1.59 \\
0.04 & 8 & 0.8 & 1.6 \\
0.05 & 8 & 0.82 & 1.59 \\
0.06 & 8 & 0.85 & 1.66 \\
0.07 & 8 & 0.88 & 1.69 \\
0.08 & 8 & 0.91 & 1.74 \\
0.09 & 8 & 0.9 & 1.7 \\
0.1 & 8 & 0.9 & 1.64 \\
\hline
\end{tabular}

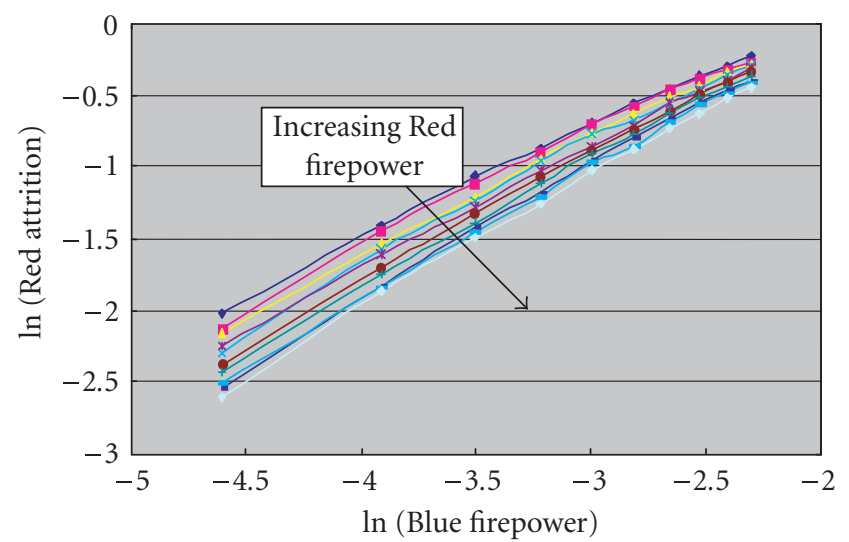

Figure 3.4. Figure 3.3 data shown on a Log-Log plot.

\section{The second term of the metamodel}

By writing (2.1) as

$$
E\left(\left|\frac{\Delta b}{\Delta t}\right|\right) \propto \Delta t^{-r(D)}
$$

for some function $r(D)$, we can focus on the second term of the metamodel. Equation (2.9) showed that the resulting power spectrum of Blue agents $P(f)$ is in the form of a power law, $P(f)=a|f|^{-(D+1)}$, where $a$ is some constant. This implies that $\log P(f)=$ $\log a-(D+1) \log |f|$. In other words, when the power spectrum is plotted on a Log-Log plot, we expect a straight line with gradient $-(D+1)$, with a value between -1 and -3 .

To test this for casualties, a variety of MANA scenarios were run and the time series representing Blue casualty data were recorded. We then looked for evidence of a power law relationship between the power spectrum of this data $P(f)$ and the frequency $f$. 
4.1. Data. The data required for this part of the work were time series of casualty data, that is, the number of agents killed at each time step. MANA can record such step-bystep data, however in our work we have used "contact data." Contact data measure the number of enemy agents that each friendly agent can see. Therefore, enemy agents can be counted more than once.

We have used contact data because there is not enough information from casualty data to calculate a good power spectrum. For example, quite often, there are no casualties for several time steps. This is because it is, for example, the companies that are modelled, not the individual men, and therefore the probability of killing an agent (a company) is small. However, we assume that every time there is contact between agents (i.e., they can detect one another), there will be casualties (perhaps only on a small scale). Therefore, contact data can be used to represent casualties. Note that when contact increases, the number of casualties is likely to increase.

4.2. Scenarios in MANA. Several MANA scenarios were used in order to analyse different types of situations. These scenarios were

(i) Western Front in World War Two [11];

(ii) the "Lanchester," "Meet," and "Swarm" scenarios mentioned earlier.

4.3. Power spectrum analysis. When using sampled data, the discrete Fourier transform approximation is used, and the squared modulus is found for each of the elements of the approximate Fourier transform. Using sampled (discrete) data rather than the "actual" continuous data means that we can only estimate the power spectrum. There is a limit to the number of data points that can be produced, as well as the length of the time step. When using the discrete Fourier transform, there are standard methods for overcoming the problems of "aliasing" and "frequency leakage" that are associated with this situation, as discussed in Press et al. [15] and Bracewell [3].

In our work we have sought to improve the estimate by

(i) averaging over a partition of the data, in which each segment has had its power spectrum taken;

(ii) using a window function to reduce leakage;

(iii) replicating MANA runs and aggregating the resulting power spectra.

As no tool existed to do all of this analysis automatically, and so that we had maximum control over the approach used, we have developed our own algorithms to carry this out. The details are given below.

4.4. Power spectrum analysis tool. The mathematical definition of the power spectrum ( $P$ as a function of frequency $f$ ) is the absolute value of the Fourier transform (FT) of the time series data $\left(c_{t}\right)$, squared. We are dealing with sampled data, and we thus used the power spectrum approximation known as the periodogram estimate, as discussed in Press et al. [15]. Firstly we define the discrete Fourier transform

$$
\mathrm{FT}_{k}=\sum_{j=0}^{N-1} c_{j} e^{2 \Pi i j k / N} \quad \text { for } k=0,1,2, \ldots, N .
$$

Thus the power spectrum approximation is $P\left(f_{k}\right)=\left|\mathrm{FT}_{k}\right|^{2}$ for $k=0,1,2, \ldots, N$. 
James Moffat et al. 9

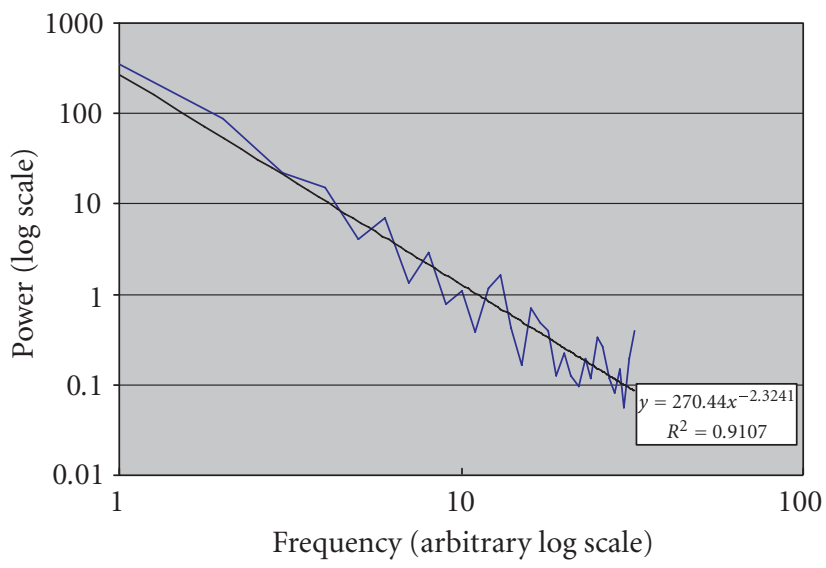

Figure 4.1. Power spectrum of contact data from Lauren's European Western Front scenario with line of best fit.

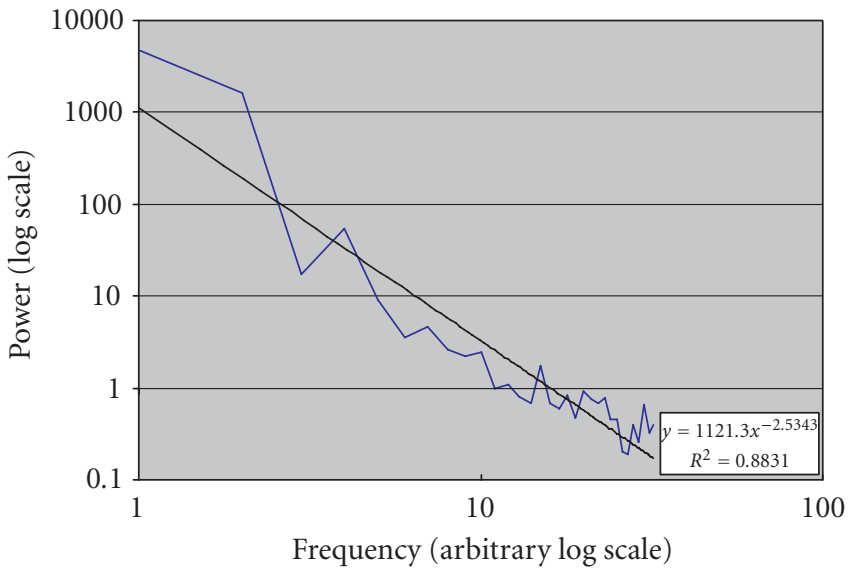

Figure 4.2. Power spectrum of casualty data from "Lanchester" scenario with line of best fit.

The periodogram estimate is then used to normalise the power spectrum such that Parseval's theorem holds (i.e., the sum of all the power terms equals the mean-squared amplitude of the transform), so that we have

$$
\begin{gathered}
\operatorname{PE}\left(f_{0}\right)=\frac{1}{N^{2}} P\left(f_{0}\right), \\
\operatorname{PE}\left(f_{k}\right)=\frac{2}{N^{2}} P\left(f_{k}\right) \quad \text { for } k=1,2, \ldots,\left(\frac{N}{2}-1\right), \\
\operatorname{PE}\left(f_{N / 2}\right)=\frac{1}{N^{2}} P\left(f_{N / 2}\right) .
\end{gathered}
$$




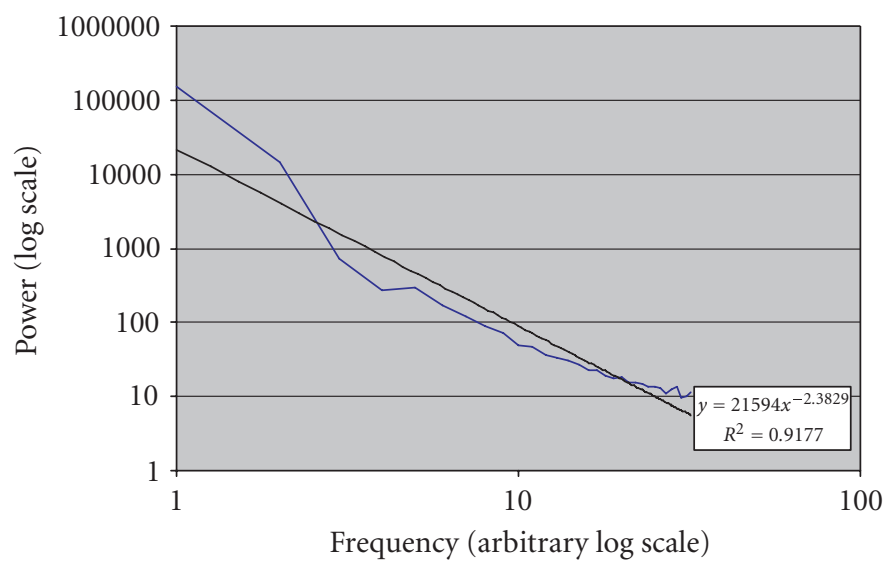

Figure 4.3. Power spectrum of contact data from "Meet" scenario with line of best fit.

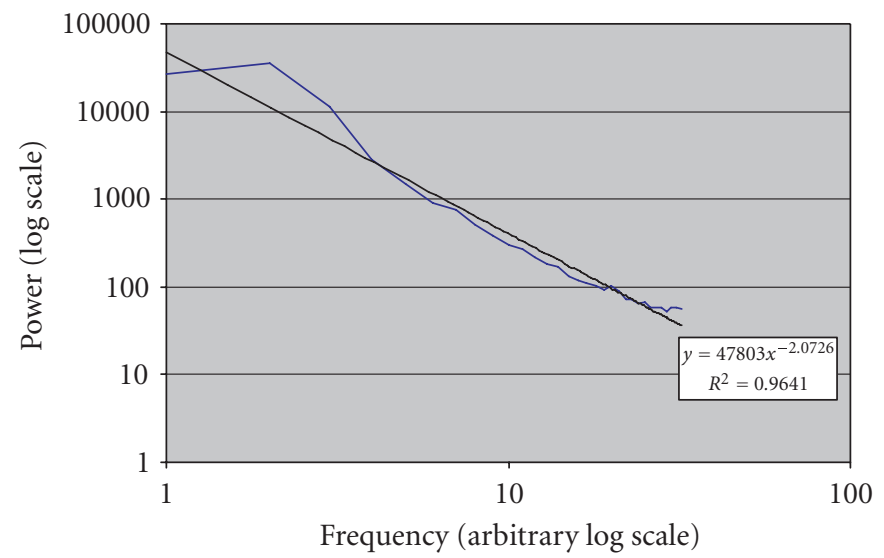

Figure 4.4. Power spectrum of contact data from "Swarm" scenario with line of best fit.

Note that the values of $P$ from points $(N / 2+1)$ to $(N-1)$ are equal to the values at the points $(N / 2-1)$ to 1 , respectively, because the input data are always real. Therefore, it is not necessary to calculate these.

To prevent "leakage," a window function can be used. This weights the input data so that the beginning and end of the time series are given less emphasis. The weighting function we considered was the Parzen window:

$$
w_{j}=1-\left|\frac{j-(1 / 2)(N-1)}{(1 / 2)(N+1)}\right| .
$$




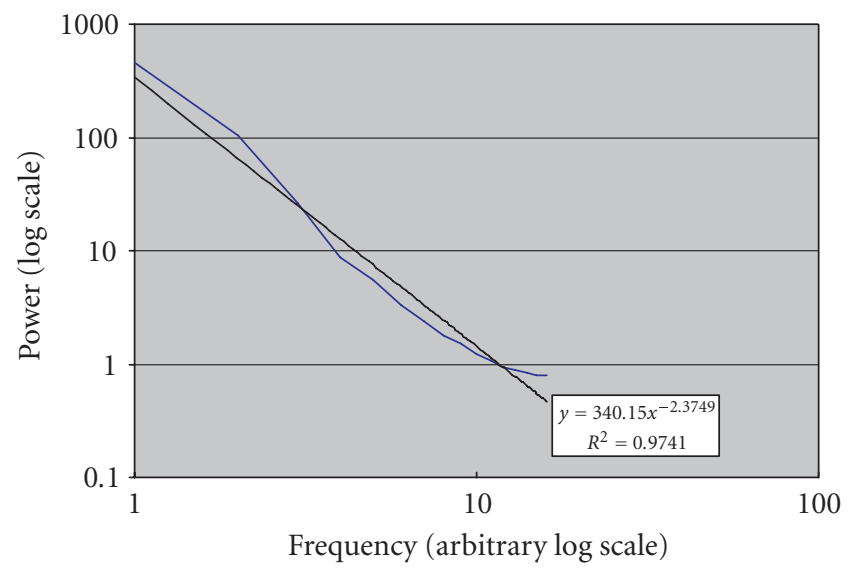

Figure 4.5. Aggregated results from 255 replications of European Western Front scenario power spectra, with line of best fit.

Therefore, the FT is calculated as

$$
\mathrm{FT}_{k}=\sum_{j=0}^{N-1} w_{j} c_{j} e^{2 \Pi i j k / N} \quad \text { for } k=0,1,2, \ldots, \frac{N}{2}
$$

To reduce the variance of the estimate of the power spectrum then calculated, data is partitioned into segments (each must have a multiple of 2 data points). The power spectrum estimate calculated from each set of data is then averaged at each frequency to increase confidence in the result. $\mathrm{K}$ segments reduce the variance of the estimate by a factor of $K$. In the results presented below, we did not use the Parzen window function (i.e., all weights are set to 1). This was because it appeared to distort the calculation of the best fit approximation to the power spectrum for our data sets.

4.5. Initial results. Four graphs follow in Figures 4.1 to 4.4. These depict the Log-Log plot of the power spectrum of the contact data from a single MANA run in each of our scenarios. The graphs illustrate (using regression analysis) that a power law (indicated by a straight line) explains $88 \%$ to $96 \%$ of the data in our experiments. In addition, the gradients of the straight lines are between -1 and -3 , as expected from our theory.

In order to reduce the variance of the estimate further, we have replicated the MANA scenarios over 200 times so that the power spectra of these runs can be averaged, to produce a better estimate. The results of this analysis are displayed in Figures 4.5 and 4.6.

It can be seen that a straight line explains $97 \%$ of the data in the aggregated case in Figure 4.5. This is a better fit that the individual replication shown in Figure 4.1, in which a straight line explained $91 \%$ of the data. This result confirms the gradient of about -2.3 that was seen in the single replication.

In Figure 4.6, 91\% of the data is now explained by a straight line, compared to $88 \%$ in the single replication. A gradient of about -2.5 is seen, which confirms the result from Figure 4.2. 


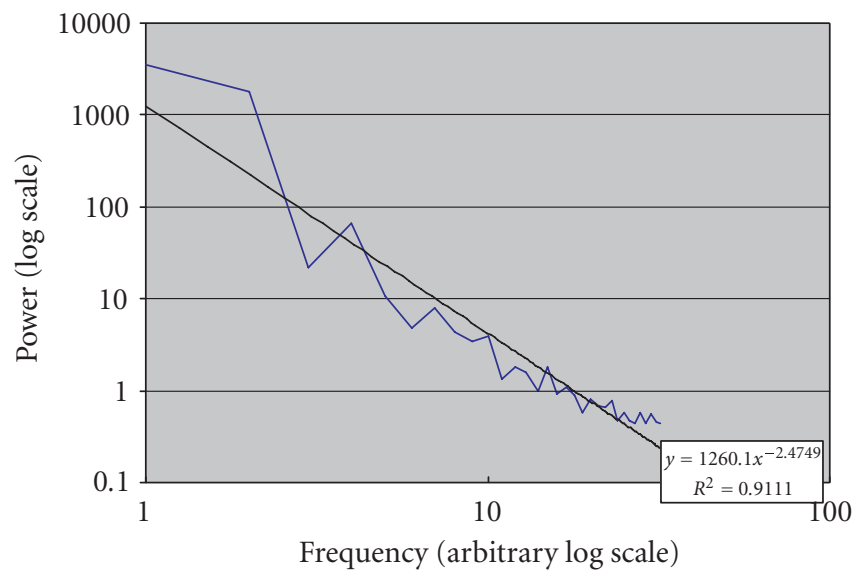

Figure 4.6. Aggregated results from 250 replications of "Lanchester" scenario power spectra, with line of best fit.

Figures 4.5 and 4.6 show that over many replications of these scenarios, a power law relationship is very clear and the gradient is still between -1 and -3 , as expected.

\section{Conclusions}

We had previously developed a theory of casualty generation which replaced the "industrial age" Lanchester equations with the fractal attrition relationship. This is more appropriate to the more self-organising types of conflict likely to occur in the "information age." Here we have shown that the predictions of this theory are consistent, in general terms, with the results of a number of scenarios investigated experimentally using the MANA agent-based simulation model of conflict.

\section{References}

[1] D. S. Alberts and R. E. Hayes, Power to the Edge, Command and Control Research Programme, US Dept of Defense, Washington, DC, 2003.

[2] G. I. Barenblatt, Scaling, Self-Similarity, and Intermediate Asymptotics, Cambridge Texts in Applied Mathematics, vol. 14, Cambridge University Press, Cambridge, 1996.

[3] R. N. Bracewell, The Fourier Transform and Its Applications, McGraw-Hill, New York, 2000.

[4] R. Darilek, W. Perry, J. Bracken, J. Gordon, and B. Nichiporuk, Measures of Effectiveness for the Information Age Army, RAND, California, 2001.

[5] A. Illachinski, Irreducible semi-autonomous adaptive combat (ISAAC): an artificial life approach to land combat, Military Operations Research 5 (2000), no. 3, 29-45.

[6] H. J. Jensen, Self-Organized Criticality, Cambridge Lecture Notes in Physics, vol. 10, Cambridge University Press, Cambridge, 1998.

[7] M. K. Lauren, A conceptual framework for understanding the power-exponent nature of empirically obtained casualty laws, DTA Report 186 NR 1376 ISSN 1174-3387, Defence Technology Agency, New Zealand, 2002.

[8] _ A fractal based approach to equations of attrition, Military Operations Research 7 (2002), no. $3,17-29$. 
[9] __ Firepower concentration in cellular automaton combat models — an alternative to Lanchester, Journal of the Operational Research Society 53 (2002), no. 6, 672-679.

[10] On the temporal distribution of casualties and determination of medical logistical requirements, DTA Report 187 NR 1377 ISSN 1175-6594, Defence Technology Agency, New Zealand, 2003.

[11] M. K. Lauren and R. Stephen, Fractals and combat modeling: using mana to explore the role of entropy in complexity science, Fractals 10 (2002), no. 4, 481-489.

[12] _ Map-aware non-uniform automata (MANA)-a New Zealand approach to scenario modelling, Journal of Battlefield Technology 5 (2002), no. 1, 27-31.

[13] J. Moffat, Complexity Theory and Network Centric Warfare, Command and Control Research Programme, US Dept of Defense, Washington, DC, 2003.

[14] - Mathematical modelling of information age conflict, to appear in Journal of Applied Mathematics and Decision Sciences.

[15] W. H. Press, S. A. Teukolsky, W. T. Vetterling, and B. P. Flannery, Numerical Recipes. The Art of Scientific Computing, 2nd ed., Cambridge University Press, Cambridge, 1992.

[16] J. G. Taylor, Lanchester Models of Warfare, Volumes 1 and 2, Military Applications Section, ORSA, Washington, DC, 1983.

[17] Project Albert, 2006, http://www.projectalbert.org.

James Moffat: Defence Science and Technology Laboratory, Policy and Capability Studies Department, Ively Road, Farnborough, Hampshire GU14 0LX, UK

E-mail address: jmoffat@dstl.gov.uk

Josephine Smith: Defence Science and Technology Laboratory, Policy and Capability Studies Department, Ively Road, Farnborough, Hampshire GU14 0LX, UK

E-mail address: jsmith@dstl.gov.uk

Susan Witty: Defence Science and Technology Laboratory, Policy and Capability Studies

Department, Ively Road, Farnborough, Hampshire GU14 0LX, UK

E-mail address: switty@dstl.gov.uk 


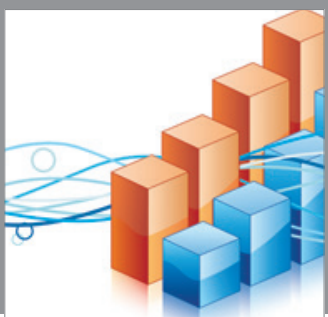

Advances in

Operations Research

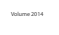

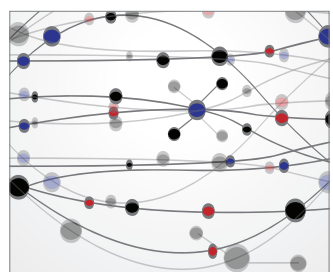

\section{The Scientific} World Journal
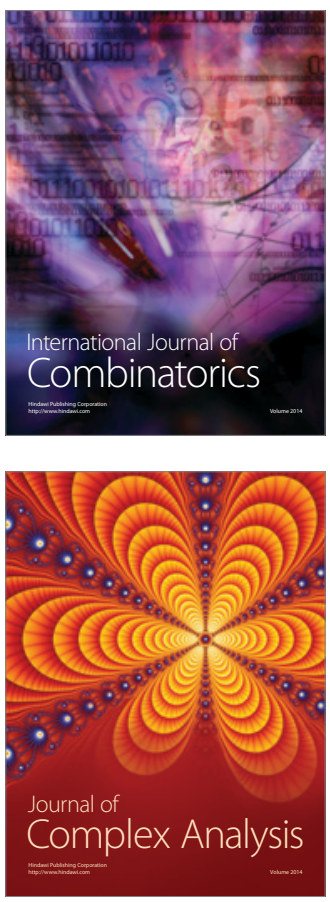

International Journal of

Mathematics and

Mathematical

Sciences
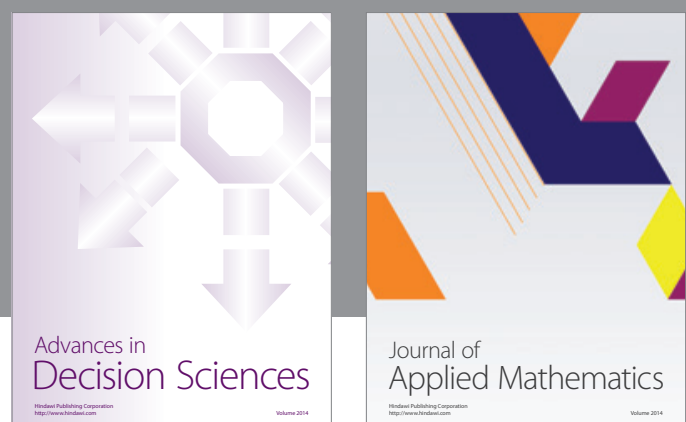

Journal of

Applied Mathematics
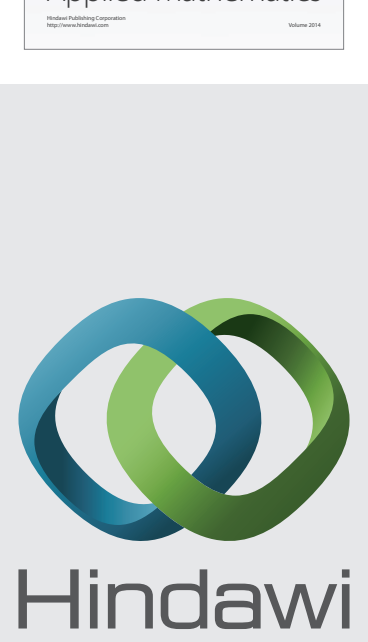

Submit your manuscripts at http://www.hindawi.com
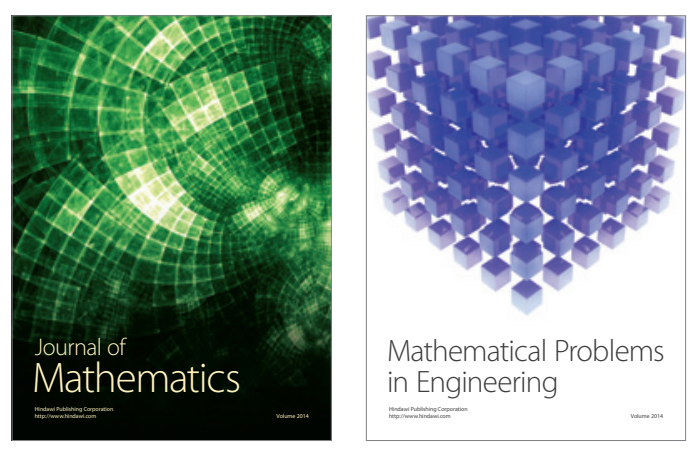

Mathematical Problems in Engineering
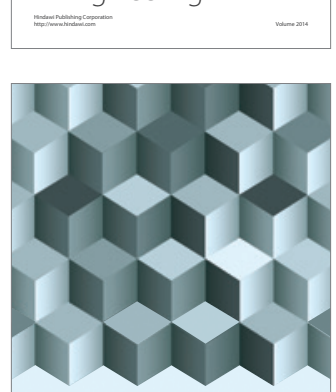

Journal of

Function Spaces
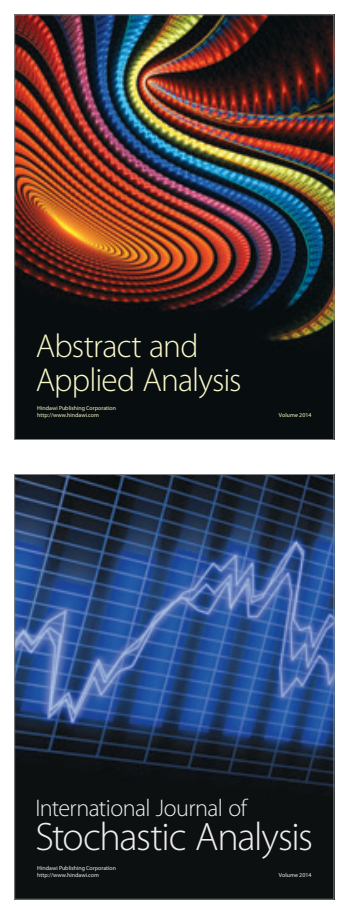

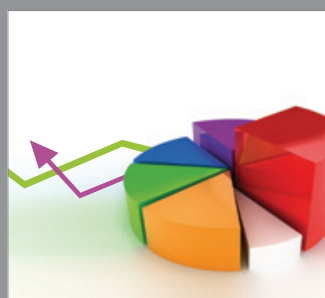

ournal of

Probability and Statistics

Promensencen
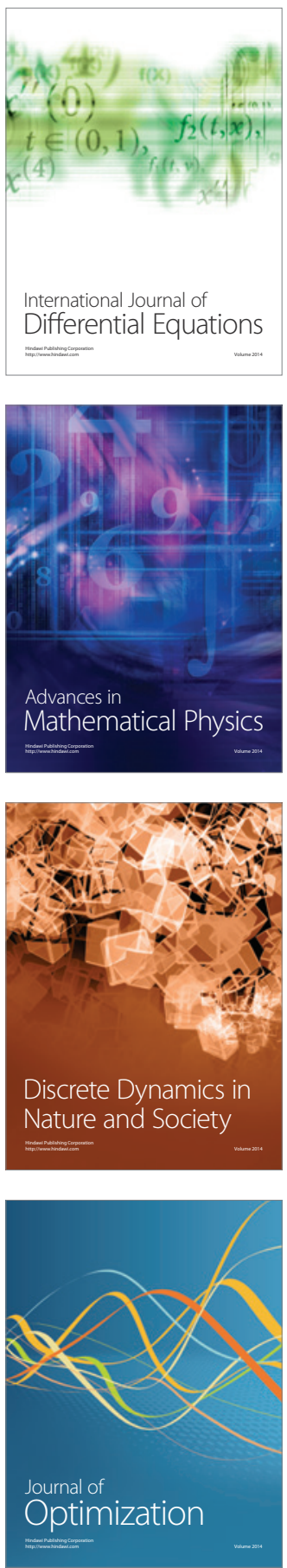